\title{
MULTI-SENSOR GEODETIC APPROACH FOR LANDSLIDE DETECTION AND MONITORING
}

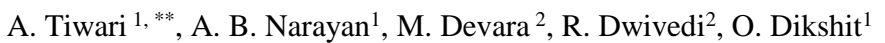 \\ ${ }^{1}$ Indian Institute of Technology, Civil Engineering - (atiwari, avadhbn, onkar)@iitk.ac.in \\ ${ }^{2}$ Motilal Nehru National Institute of Technology, GIS Cell- (rgi1703, ramjid) @ mnnit.ac.in
}

Commission V, SS: Disaster Monitoring, Damage Assessment and Risk Reduction

KEY WORDS: Landslide monitoring, geodetic techniques, LiDAR, GNSS network adjustment

\begin{abstract}
:
The lesser Himalayan region is mostly affected by landslide events occurring due to rainfall, steep slopes and presence of tectonic activity beneath, causing loss of life and property. Some critical zones in the region have encountered recurring landslides over the past and need careful investigation for better planning and rescue operations. This research work presents a geodetic framework comprising multiple sensors to monitor the Sirobagarh landslide in Uttarakhand, India, which is affected by recurring landslides. Three field visits were made to this site for geodetic data collection using Terrestrial Laser Scanner (TLS), Global Navigation Satellite System (GNSS) and Robotic Total Station (RTS). Co-registration and vegetation removal of the TLS scans corresponding to the three visits resulted in generation of three Digital Elevation Models (DEM), which were differenced to estimate temporal movement of the landslide scarp. DEM differences indicate subsidence of the landslide scarp with vertical displacement values ranging from -0.05 to $-5.0 \mathrm{~m}$. Rainfall induced debris flow is one of the prominent reason for large displacement magnitude $(\sim 5 \mathrm{~m})$ in the upper landslide scarp. Horizontal displacement estimates obtained by geodetic network analysis of six GNSS stations installed on the study site show movement towards the Alaknanda river. The maximum horizontal and vertical displacement values for the GNSS stations were $0.1305 \mathrm{~m}$ and $-2.1315 \mathrm{~m}$ respectively. Similar pattern is observed by displacement measurements of RTS target reflectors installed on a retaining wall constructed to arrest the debris flow approaorching the National Highway. The displacement estimates obtained from the sensors applied in this study indicate subsidence of the landslide scarp and surroundings. More time series observations can provide better understanding of the overall deformation process.
\end{abstract}

\section{INTRODUCTION}

\subsection{Landslide detection and analysis}

Analysing landslide events requires assessment of data collection, processing and accuracy related to time series observations. Modern geodetic techniques, such as Terrestrial Laser Scanning (TLS), Global Navigation Satellite System (GNSS), Close-range Photogrammetry (CRP), Robotic Total Station (RTS) survey, Digital levelling, etc. help in surveying areas affected by such events, at different spatial scales. Some of the aforementioned techniques are helpful in surveying small landslides, while others can help in surveying larger area, but at the cost of lower data density. Geo-location information obtained from these sensors/techniques has found widespread application in temporal analysis of areas prone to deformation events such as landslides, slope instabilities, land subsidence and upliftment, etc. (Tzur et al., 1996; Teke et al., 2008; Syahmi et al., 2011; Jaboyedoff et al., 2012; Fuhrmann et al., 2015).

TLS point clouds of different times need to be co-registered and transformed to one coordinate system (e.g. using affine transformation) for change detection studies. Generally, adjusted GNSS station coordinates are used to register TLS point clouds. However, tie points obtained from setting up target points on the field, or manually selecting tie points common to the scans are other alternatives that help in coregistration, depending on the geographical accessibility and geological complexity of the site under investigation (Jebur et al., 2014). Apart from co-registration, non-ground objects like trees, bushes, shrubs, etc. are extracted and removed from the point cloud for DEM generation, which can be used to compute temporal movement.

GNSS based deformation analysis generally involves (i) establishment of a network of GNSS stations, (ii) their adjustment, (iii) transformation to common datum and (iv) differencing for displacement detection. The GNSS stations should preferably be well distributed and free from obstructions, facing clear sky and environmental conditions (Tzur et al., 1996; Setan and Singh, 2001; Cederholm, 2003; Dwivedi and Dikshit, 2011; Singh et al., 2016). Statistical post analysis is further used to find the outlier observations from the network, and to test the accuracy of the displacement estimates. GNSS horizontal displacement rates are more reliably quantified and interpret compared to vertical estimates. The vertical displacement values are generally corrected with the help of precise levelling operations (Ching et al., 2011; Qin et al., 2017).

Field surveys related to slope instability studies require survey of inaccessible areas for high resolution DEM generation. For GNSS, such surveys can be time intensive and infeasible. Such areas can be surveyed from a distance of 10-200 m with TLS, resulting in dense point clouds (millions of points $/ \mathrm{m}^{2}$ ) and high resolution DEMs, which can be used for measuring surface movement and volume change (Denora et al., 2011; Barbarella and Fiani, 2013; Syahmi et al., 2011; Wan Aziz et al., 2012;

\footnotetext{
${ }^{*}$ Corresponding author
} 
Wang et al., 2017). However, in case of large areas, TLS survey has issues related to occlusion and co-registration errors.

\subsection{Objective}

The objective of this study is to detect time series surface displacement of a landslide prone 'Sirobagarh region' using TLS, GNSS and RTS field surveys.

\subsection{Study area}

Sirobagarh $\left(30.24^{\circ} \mathrm{N} 78.89^{\circ} \mathrm{E}\right)$ is situated on the left bank of the Alaknanda river, about $12 \mathrm{~km}$ from Srinagar, accessible via a national highway NH58. Since 1960s, landslide activity has been reported in the area, which very often obstructs the National Highway 'NH58'. The area is considered as highly prone to landslides occurring due to local geological conditions and rainfall. A retaining wall is constructed by the Public Works Department (PWD) to arrest debris approaching NH58. In addition, a village named 'Chhatikhal' is located $18 \mathrm{~m}$ above the landslide peak, and is vulnerable to further damages due to recurring slides. The study site has a history of landslide events and is highly susceptible to slide in near-future. A reconnaissance survey was initially conducted to find out appropriate locations for the TLS scans, GNSS stations and RTS stations and reflectors. TLS is used for scanning the landslide scarp, while GNSS and RTS observations establish a geodetic network in the area surrounding the landslide. Figure 1 shows an oblique Google earth view of the study area and the location of the geodetic instruments used during the field surveys. The black rectangle shows target reflectors installed on the retaining wall for RTS observations. GNSS stations are names GPS $\mathrm{i}, i \rightarrow 1$ to 6. TLS scans and some of the GNSS stations had to be set up alongside NH58 for better visibility and network geometry.

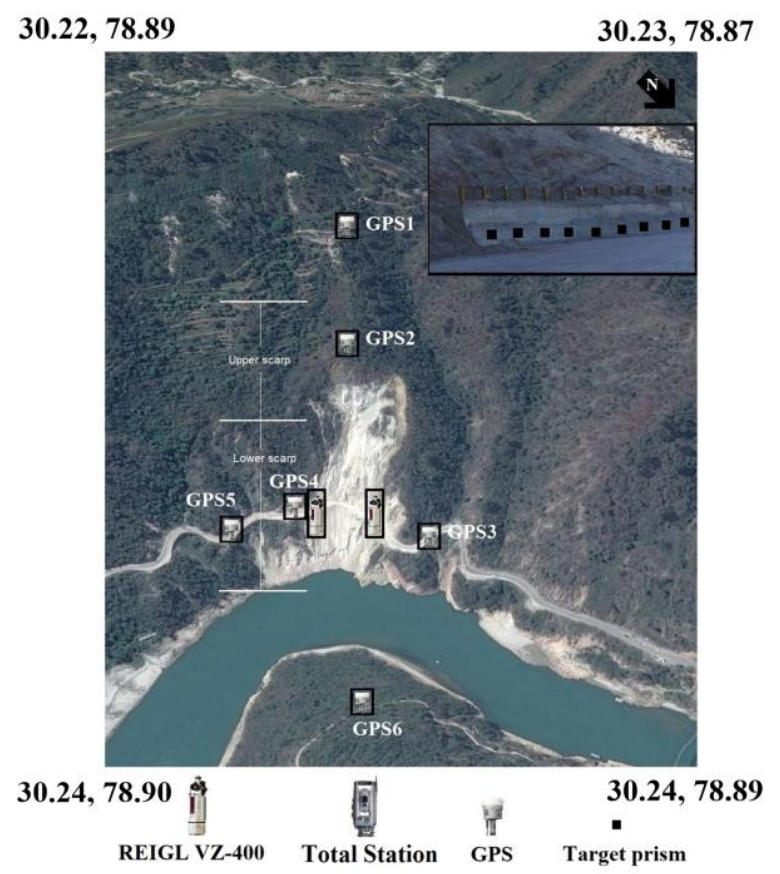

Figure 1. An oblique Google earth view of the study area. Large black rectangular box shows the retaining wall and the installed target reflectors.
Three field visits, over a period of 17 months were made to the study site (17 Oct 2015 to 18 Mar 2017), accounting for the yearly variations in rainfall and environmental conditions. Each visit was conducted for five days. The next section describes the methodology adopted in this research work.

\section{METHODOLOGY}

\subsection{TLS survey}

The raw TLS data scans obtained from laser scanning were processed to generate a point cloud. The overall process involved co-registration of different scans and vegetation removal. Initially, all the points of a scan $\operatorname{Scan}_{i j}(i$ and $j$ denote the scan number and visit number respectively) were in a local coordinate system, usually called the local scanner coordinate system with the scan position as the origin of such a system. Since different scans had scan positions with different origins and axes orientation, a coordinate transformation was required to align these scans to a common reference system. One of the ways to do this was to set up a group of GNSS points within the scan area and select points present in all the scans as ground control points (GCPs). The common set of GCPs would then be used to transform the scanner coordinate system into the global earth centered earth fixed (ECEF) system using affine transformation. Transformation of scanner coordinates to ECEF coordinates is shown in equation (1), where the translation vector $T$ is the displacement required to shift the origin of scanner coordinates to that of the other coordinate systems, $R_{x}(\omega), R_{y}(\phi)$ and $R_{z}(\kappa)$ are the rotation matrices along the $\mathrm{X}, \mathrm{Y}$ and $\mathrm{Z}$ axes, with $\omega, \phi$ and $\kappa$ being roll, pitch and yaw respectively.

$$
\begin{gathered}
{\left[\begin{array}{c}
X \\
Y \\
Z \\
1
\end{array}\right]=\left[R_{x}(\omega) R_{y}(\phi) R_{z}(\kappa)\right] \times[T(x, y, z)]\left[\begin{array}{c}
x \\
y \\
z \\
\text { projected } \\
\text { or global } \\
1
\end{array}\right]} \\
\sum_{X_{T L S}}=R \sum_{X_{G C P}} R^{T}
\end{gathered}
$$

The covariance matrix of this transformation is given by equation (2), where $\sum_{X_{T L S}}$ and $\sum_{X_{G C P}}$ are the covariance matrices of the transformed ECEF point cloud coordinates and that of GCPs respectively. One disadvantage associated with this method is the error propagated from GCPs to the transformed coordinates of the cloud points. Instead of converting all the cloud points into the ECEF system, in this study, coordinates of all the scans were transformed to the reference system of one of the scans, i.e. to $\operatorname{Scan}_{11}$. This method did not require GCPs, points common in all the scans were selected manually and equation (1) was used for transformation. Error in the selection of the manual tie points would reflect the co-registration error, but this method aided in getting rid of the error propagated from the GCPs. 
Further, to avoid elevation computation errors, vegetation removal was done. Tree and bush like features were extracted using segmentation and convex hull was later employed to detect boundaries of these features (Parida and Rajan, 2017). Consequently, the features belonging to vegetation were removed from the point cloud. After vegetation removal, DEMs corresponding to the three visits were generated and subsequently differenced to detect the temporal surface movement.

\subsection{GNSS geodetic network adjustment}

A geodetic network of six GNSS stations was established during the three field visits for displacement detection and analysis. A relative network was set up with all the points lying within the deformable object, and one of the stations (GPS3) acting as a base station (Aydin, 2014). The detection procedure involved (i) network compatibility test, (ii) transformation into a common datum, (iii) global and local congruency tests and (iv) displacement detection. The detection procedure is shown in Figure 2. Initially, networks of different epochs (each visit here refers to one epoch, hence a total of three epochs) were individually adjusted, which involved adjustment of the coordinates of each station with respect to the master (GPS3) using least squares adjustment (Burkholder, 2004). Displacement vectors between two epochs $k$ and $k+1$ were then calculated by taking differences of the adjusted coordinates, as shown in equation (3). The individually adjusted networks were tested for compatibility. Once found compatible, the networks were simultaneously adjusted by transforming these to a common reference datum using $S$ transformation (Cai et al., 2008; Aydin, 2014; Setan and Singh, 2001). Subsequently, deformation estimates were also transformed, as shown in equation (4), where and are the transformed and the original deformation vectors respectively, and are the transformed and the original deformation covariance matrices respectively and $S$ is the S-transformation matrix.

$$
d_{i j}=G P S_{j}^{k+1}-G P S_{i}^{k}=\left[\begin{array}{c}
x_{G P S_{j}^{k+1}}-x_{G P S_{i}^{k}} \\
y_{G P S_{j}^{k+1}}-y_{G P S_{i}^{k}} \\
z_{G P S_{j}^{k+1}}-z_{G P S_{i}^{k}}
\end{array}\right]
$$

Global congruency test was applied to test the stability of the complete network, using the test statistic shown in equation (4), where $\hat{\sigma}_{0_{k+1}}^{2}$ and $\hat{\sigma}_{0_{k}}^{2}$ represent the aposteriori reference variances for adjusted networks of epochs $k+1$ and $k$ respectively, $\alpha$ denotes the significance level, $d f_{k+1}$ and $d f_{k}$ are the degrees of freedom corresponding to epochs $k+1$ and $k$ respectively. The global test failed $\left(T_{v a r}>F_{\text {tabulated }}\right)$, hence local congruency test was applied to each point in the network which continued till all the network points were found to be stable. Once the network contains all stable points, magnitude of the deformation vectors were compared with a threshold value. If the magnitude was larger than the threshold, the point was said to be deformed, otherwise considered stable over the epochs
(Caspary, 1990; Cai et al., 2008; Cederholm, 2008; Setan and Singh, 2001).

$$
T_{v a r}=\frac{\hat{\sigma}_{0_{k+1}}^{2}}{\hat{\sigma}_{0_{k}}^{2}} \sim F\left(\alpha, d f_{k+1}, d f_{k}\right)
$$

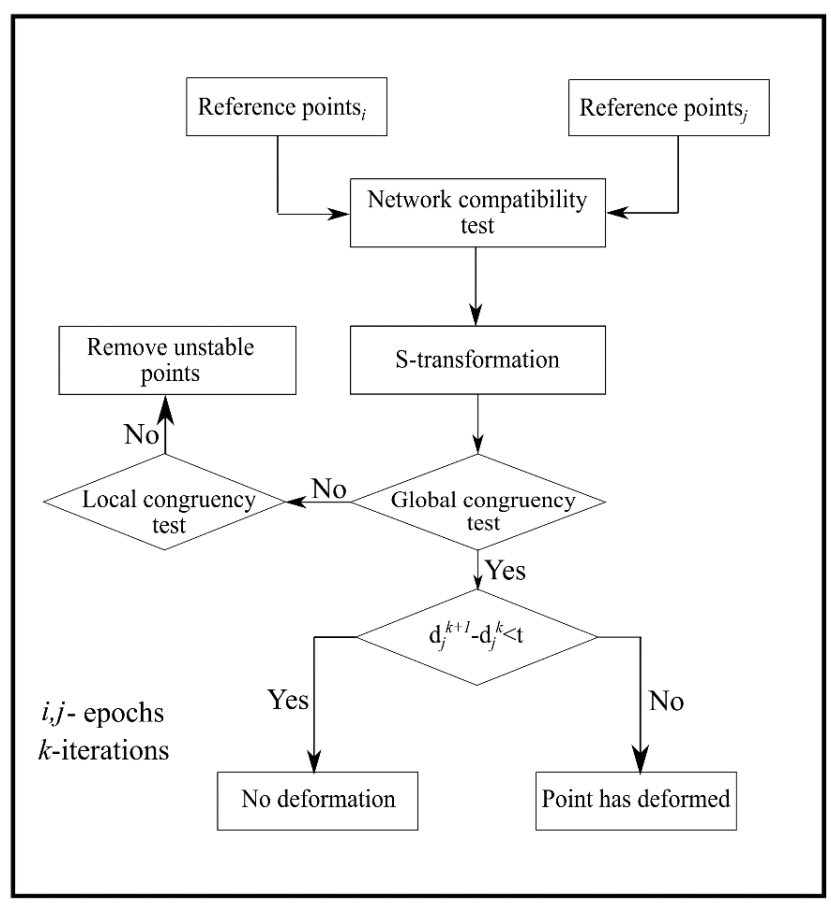

Figure 2. GNSS geodetic network processing for displacement detection and analysis.

\subsection{RTS temporal change detection}

In order to find out the temporal movement of the retaining wall located at the lower scarp of the landslide, 13 target reflectors were installed (Figure 1). But due to rainfall and debris flow, only nine target reflectors could survive for the time period of the three field surveys. Two relatively stable points, one situated in a temple and the other on the other side of the river were selected as backsight station (BS) and instrument station (IS) respectively. The RTS backsight (BS) and intermediate sight (IS) stations were setup nearby the GNSS base station and the coordinates of these stations were also adjusted and transformed to WGS 84 coordinates using the GNSS base station coordinates during the three visits. Subsequently, coordinates of the target points (TPs) installed on the retaining wall were measured using the RTS set up on the IS. Finally, coordinates of the TPs were differenced over the epochs to detect the displacement of the retaining wall. Accuracy of the vertical measurements can be computed using root mean square error (RMSE), as shown in equations (5), (6) and (7), where $\mathrm{RMSE}_{\mathrm{Z}}$ denotes the error in elevation computation, $i$ represents the point (target reflector) under investigation, $Z_{\text {epoch }_{\mathrm{k}+1}}^{\mathrm{i}}$ and $Z_{\text {epoch }_{\mathrm{k}}}^{\mathrm{i}}$ represent the elevation of the point at epochs $k+1$ and $k$ respectively, and $n$ is the number of points. Similarly, RMSE values for the $X$ and $Y$ coordinates can also be computed. 


$$
\begin{aligned}
& \operatorname{RMSE}_{\mathrm{Z}}=\sqrt{\sum_{\mathrm{k}=1}^{3} \sum_{\mathrm{i}=1}^{\mathrm{n}} \frac{\left(\mathrm{Z}_{\text {epoch }_{\mathrm{k}+1}}^{\mathrm{i}}-\mathrm{Z}_{\text {epoch }_{\mathrm{k}}}^{\mathrm{i}}\right)^{2}}{\mathrm{n}}} \\
& \operatorname{RMSE}_{\mathrm{X}}=\sqrt{\sum_{\mathrm{k}=1}^{3} \sum_{\mathrm{i}=1}^{\mathrm{n}} \frac{\left(\mathrm{X}_{\text {epoch }_{k+1}}^{\mathrm{i}}-\mathrm{X}_{\text {epoch }_{\mathrm{k}}}^{\mathrm{i}}\right)^{2}}{\mathrm{n}}} \\
& \operatorname{RMSE}_{\mathrm{Y}}=\sqrt{\sum_{\mathrm{k}=1}^{3} \sum_{\mathrm{i}=1}^{\mathrm{n}} \frac{\left(\mathrm{Y}_{\text {epoch }_{k+1}}^{\mathrm{i}}-\mathrm{Y}_{\text {epoch }_{\mathrm{k}}}^{\mathrm{i}}\right)^{2}}{\mathrm{n}}}
\end{aligned}
$$

\section{RESULTS AND DISCUSSIONS}

\subsection{TLS survey results}

Differences of the TLS derived DEMs (DEM1, DEM2 and DEM3) corresponding to the three field visits are shown in Figure 6. It is observed that the landslide scarp had undergone subsidence between epochs 1-2 and epochs 1-3. Most of the points indicate downward movement, with displacement values close to -0.5 to $0.5 \mathrm{~m}$ for most of the points. The points present in the upper landslide scarp show higher vertical displacement magnitude with values ranging from $0.5-5 \mathrm{~m}$, probably due to the effect of debris flow. DEM differences for the differences in epoch interval 1-3 contain greater number of points in the landslide scarp with subsidence values $\sim 5 \mathrm{~m}$ compared to that of epoch interval 1-2. One of the likely reasons for large subsidence values can be the occurrence of rainfall in the area during the epoch interval 2-3, which increased the rate of debris flow, causing higher displacement.

\subsection{GNSS geodetic network analysis}

The GNSS geodetic network analysis results for the six GNSS stations setup surrounding the landslide scarp (covering $500 \times 600 \mathrm{~m}^{2}$ ) show that the point GPS2 had moved significantly with a horizontal and vertical movement of $0.1305 \mathrm{~m}$ and $2.1315 \mathrm{~m}$ respectively between epochs 1 and 3. GPS2 was situated in the upper scarp of the landslide and was affected by the debris flow, which can be a reason for a large magnitude of vertical deformation. However, for the other stations also, a vertical deformation of $\sim 20 \mathrm{~cm}$ was detected, giving an indication of subsidence in the area. Figure 4 shows the horizontal and vertical displacement estimates obtained for the GNSS network points, where the yellow coloured arrows represent the horizontal movement and the color of the dots indicate vertical movement. The horizontal estimates show movement towards the Alaknanda river in the landslide slope direction which is situated below the landslide. Further, the vertical movement for points other than the base station show subsidence. From the results mentioned above, it is inferred that the area surrounding the landslide area underwent subsidence over the time period with movement in the direction of the landslide slope.
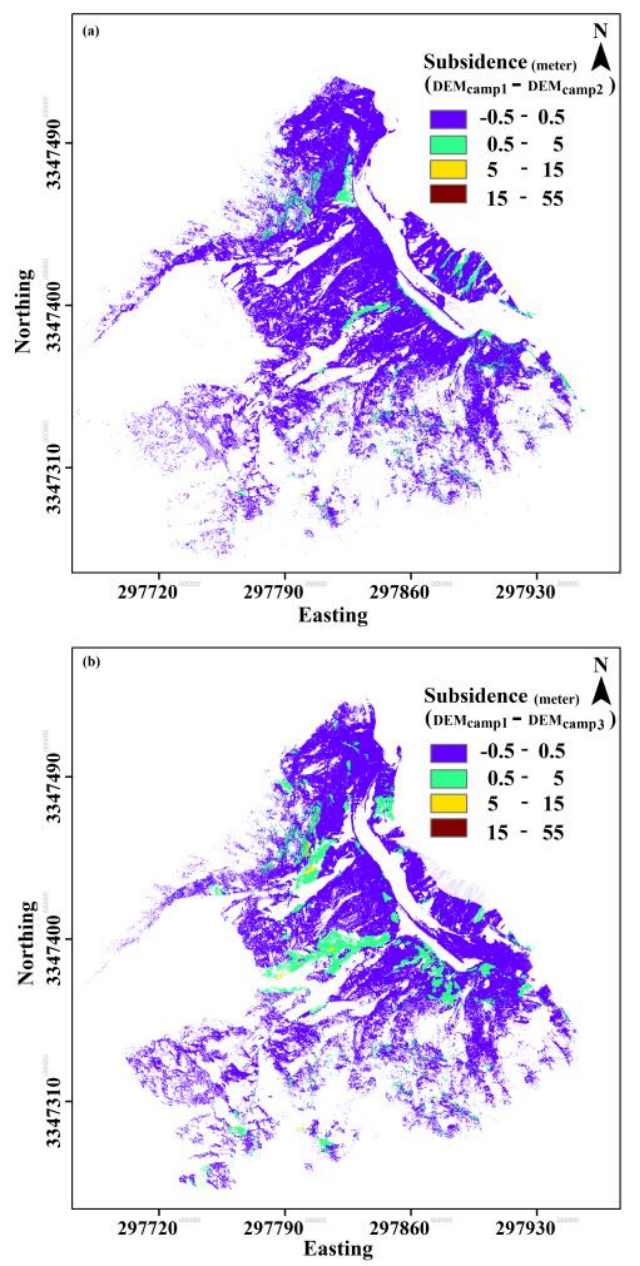

Figure 3. TLS DEM differences for (a) epoch difference 1-2 and (b) epoch difference 1-3
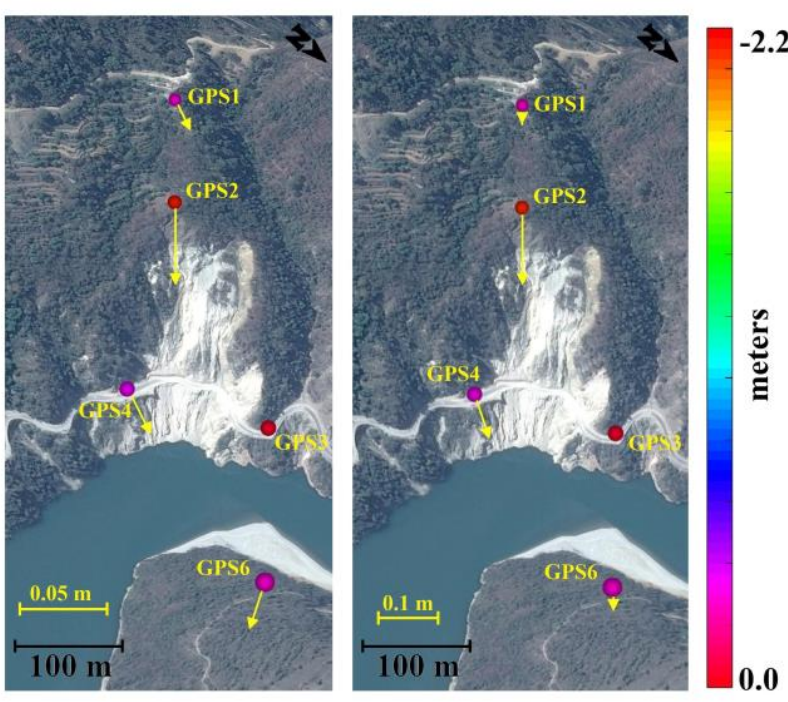

Figure 4. GNSS horizontal and vertical displacement estimates between epochs (a) 1-2 and (b)1-3 for the Sirobagarh site. Black scale bar denotes the image scale, whereas the yellow coloured shows scale for the horizontal displacement vectors. Yellow arrows show horizontal movement and the color of the dots show vertical movement. 


\subsubsection{RTS survey of the retaining wall}

Target reflectors installed on the retaining wall were sighted using the RTS instrument during all the visits. However, during the second and third visit, only 11 and 9 reflectors common to visit 1 could survive due to dislocation of other targets. It was found from the GNSS network analysis that BS and IS had undergone change during the three visits and hence, corrections were applied on the BS and IS coordinates to eliminate the surface movement bias. 3D coordinates of the target reflectors corresponding to the three trips were then computed and subtracted to find change in the retaining wall during epochs 1 2 and 1-3. Figure 5 shows the displacement of the target reflectors over epochs 1-2 and 1-3, where the horizontal displacement of the reflectors is towards the river while the vertical movement shows subsidence. The 3D displacement pattern corresponds well with that obtained using GNSS for points located in the landslide surroundings. Table 1 shows the RMSE values of $\mathrm{X}, \mathrm{Y}, \mathrm{Z}$ coordinates for epoch differences 1-2 and $1-3$.
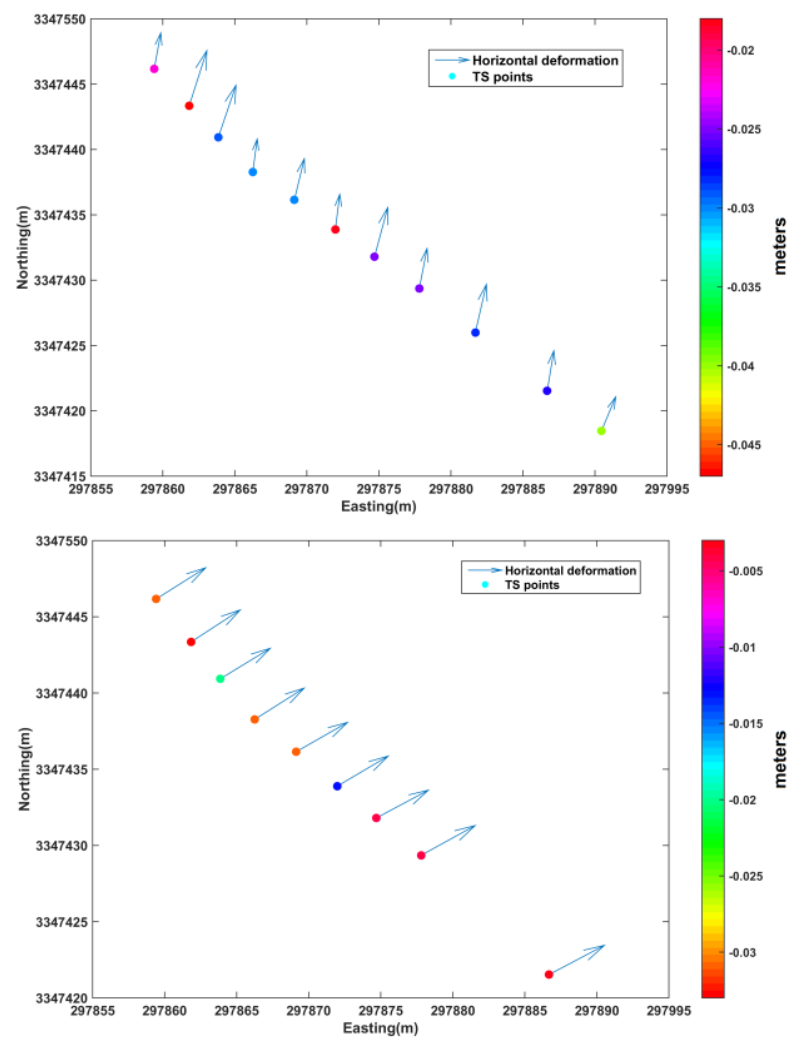

Figure 5. Displacement of target reflectors installed on the retaining wall during epochs 1-2 and 1-3.

Table 1. RMSE values RTS change measurement in X,Y,Z coordinates during three visits.

\begin{tabular}{|l|l|l|l|}
\hline ED $^{*}$ & $\mathrm{X}$ & $\mathrm{Y}$ & $\mathrm{Z}$ \\
\hline 12 & 0.0103 & 0.0427 & 0.0302 \\
\hline 13 & 0.5493 & 0.3049 & 0.0226 \\
\hline
\end{tabular}

Vertical displacement estimates obtained from the three different surveying instruments detect subsidence over the landslide and its surroundings. In case of TLS, most of the points show negative movement during the three field surveys. Apart from high magnitude movements $(\sim 5 \mathrm{~m})$ for some points, vertical movements ranged from -0.05 to $-0.5 \mathrm{~m}$ (Figure 3 ). With the GNSS network points, a maximum vertical displacement of $-2.1315 \mathrm{~m}$ was detected for GPS2 located just above the upper landslide scarp. However, other points also detected movements near to $20 \mathrm{~cm}$. The undulating terrain with presence of obstructions due to trees and hills, along with clouds and strong wind introduced unfavorable surveying conditions, which may have contributed to errors in the GNSS observations, in addition to the uncertainty involved in GNSS vertical positioning. RTS observations, similar to TLS and GNSS, detect downward movement of the retaining wall. Horizontal displacement form GNSS and RTS indicate movement towards the Alaknanda river. With similarity in pattern of horizontal and vertical displacement detected from the three sensors, it is inferred that the study site underwent subsidence, with slide movement towards the Alaknanda river situated below the landslide.

\section{CONCLUSION}

In this research work, a multi-sensor approach for landslide detection encompassing TLS, GNSS and RTS surveys was applied on a severely affected landslide prone site known as Sirobagarh landslide, which lies in lesser Himalayan region. TLS derived DEMs generated for the three epochs detected vertical displacements ranging from -0.5 to $-5 \mathrm{~m}$ for majority of points, which indicate subsidence over the landslide scarp. The network of GNSS geodetic network with six GNSS stations set up in the landslide surroundings showed horizontal movement towards the Alaknanda river (maximum value $0.1305 \mathrm{~m}$ ) and downward vertical displacement (maximum subsidence value $2.1315 \mathrm{~m}$ ). In addition to the uncertainty involved in vertical positioning, high magnitude of vertical displacements detected from GNSS observations may contain contributions other than surface subsidence due to the geological and atmospheric conditions prevailing at the time of survey, which include characteristics like undulating terrain, presence of obstructions due to trees and hills, along with clouds and strong wind at the time of data collection. Further, target prism reflectors installed on a retaining wall located at the toe of the landslide showed similar horizontal and vertical movement pattern as obtained by GNSS surveys. The obtained displacement estimates from TLS DEM differencing, GNSS network deformation estimation and RTS temporal change measurement and their separate statistical analysis indicate subsidence in the landslide scarp and near surroundings, apart from a horizontal movement towards the Alaknanda river over a duration of 17 months (the three epoch intervals). More time-series observations, along with application of more sensors can provide better insights of the ongoing subsidence in the study site. This approach can also be used to investigate deformation events at other sites similar in characteristics.

\section{ACKNOWLEDGEMENTS}

This research work is funded by the NRDMS, Department of Science and Technology (DST), India.

\section{REFERENCES}

Aydin, C., 2014. Geodetic deformation analysis: Short lecture notes for graduate students, YTU-Geodesy Division, Istanbul. 
Barbarella, M., and Fiani, M., 2013. Monitoring of large landslides by Terrestrial Laser Scanning techniques: field data collection and processing, European Journal of Remote Sensing, 46(1), 126-151.

Burkholder, E.F., 2005. Magical Least Squares - or When is one Least Squares adjustment better than another?, NMSU Department of Surveying Engineering, Las Cruces.

Caspary, W.F., Haen, W., Borutta, H., 1990. Deformation analysis by statistical methods, Technometrics, 32(1), 49-57.

Cai, J., Wang, J., Wu, J., Hu, C., Grafarend, E., Chen, J., 2008. Horizontal deformation rate analysis based on Multiepoch GNSS Measurements, Journal of Surveying Engineering-ASCE, 134 (4), 132-137.

Capilla, R.M., Berne, J.L., Martin, A., Rodrigo, R., 2016. Simulation case study of deformations and landslides using real-time GNSS precise point positioning technique, Geomatics, Natural hazards and Risk, 7 (6), 1856-1873.

Castagnetti, C, Bertacchini, C., Corsini, A., Capra, A., 2013. "Multi-sensors integrated system for landslide monitoring: critical issues in system setup and data management", European Journal of Remote Sensing, 46 (1), 104-124.

Cederholm, P., 2003. Deformation analysis using confidence ellipsoids, Survey Review, 37 (287), 31-45.

Ching, K.E., Hsieh, M.L., Johnson, K.M., Chen, K.H., Rau, R.J., Yang, M., 2011. "Modern vertical deformation rates and mountain building in Taiwan from precise leveling and continuous GPS observations, 2000-2008 “, Journal of Geophysical Research, 116, DOI: 10.1029/2011JB008242.

Denora, D., Romano, L., Cecaro, G., 2011. Terrestrial laser scanning for the Montaguto landslide (Southern Italy), Proceedings of the Second World Landslide Forum, 3-7 October 2011, Rome.

Dorota, A., Brzezinska, G., Toth, C., Wu, T.H., Shakoor, A., 2015. Probabilistic Use of LiDAR Data to Detect and Characterize Landslides, The Ohio State University.

Dwivedi, R., Dikshit, O., 2013. A comparison of particle swarm optimization (PSO) and genetic algorithm (GA) in second order design (SOD) of GPS networks, Journal of Applied Geodesy, 7 (2).

Farolfi, G. and Ventisette, C., 2017 Strain rates in the Alpine Mediterranean region: insights from advanced techniques of data processing. GPS Solutions, 21(3), pp.1027-1036.

Fressard, M., Macquire, O., Thiery, Y., Davidson, R., Lissak, C., 2016. Multi-method characterisation of an active landslide: Case study in the Pays d'Auge plateau (Normandy, France), Geomorphology, 270, 22-39.

Fuhrmann, T., Cuenca, M.C., Knopfler, A., Van Leigen, F.J., Mayer, M., Westerhaus, M., Hanssen, R., Heck, B., 2015. Combining InSAR, Levelling and GNSS for the estimation of 3D surface displacements, Fringe 2015 Workshop', Frascati, Italy, ESA SP-731, 21-27 March, 2015.
Jaboyedoff, M., Oppikofer, T., Abellan, A., Derron, M.H., Loye, A., Metxzger, R., Pedrazzini, A., 2012. Use of LIDAR in landslide investigations: a review, Natural Hazards, 61, 5-28.

Jebur, M.N., Pradhan, B., Tehrani, M.S., 2014. Optimization of landslide conditioning factors using very high-resolution airborne laser scanning (LiDAR) data at catchment scale, Remote Sensing of Environment, 152, 150-165.

Kornejady, A., Ownegh, M., Bahremand, A., 2017. Landslide susceptibility assessment using maximum entropy model with two different data sampling methods, Catena, 152, 144-162.

Lollino, G., Pasculli, A., Sciarra, N., Baldo, M., Giordan, D., 2007. An integrated methodology based On Lidar, GNSS And Photogrammetric Surveys applied to a large Landslide in San Martino Sulla Marruccina (Central Italy), Proceedings of First North American Landslide Conference, 23.

Parida, G., and Rajan, K.S., 2017. Localized segment based processing for automatic building extraction from Lidar data, International Archives of the Photogrammetry, Remote Sensing and Spatial Information Science, 42(8), Hannover, Germany.

Qin, S., Wang, W., Song, S., 2017. "Comparative study on vertical deformation based on GPS and leveling data", Geodesy and Geodynamics, 9, 115-120.

Rawat, M.S., Uniyal, D.P., Dobhal, R., Joshi, V., Rawat, B.S., Bartwal, A., Singh, D., Aswal, A., 2015. "Study of landslide hazard zonation in Mandakini Valley, Rudraprayag district, Uttarakhand using remote sensing and GIS", Current Science, 109 (1).

Setan, H., Singh, R., 2001. Deformation analysis of a geodetic monitoring network, Geomatica, 55(3).

Singh, V., Dwivedi, R., 2016. First-Order Design of GPS Networks Using Particle Swarm Optimization, ASCE Journal of Surveying Engineering, 142 (3).

Syahmi, M.Z., Wan Aziz, W.A., Zulkarnaini, M.A., Anuar, A., Othman, Z., 2011. The Movement Detection on the Landslide Surface by Using Terrestrial Laser Scanning, 2011 IEEE Control and System Graduate Research Colloquium.

Teke, K., Yalkincaya, M., Konak, H., 2008. Optimization of GNSS networks for landslide areas, Fresenius Environmental Bulletin, 17(8).

Tzur, G.E., Papo, H.B., 1996. Optimization of GNSS networks by linear programming, Survey Review, 33(62), 537-545.

Wan Aziz, W.A., Syahmi, M.Z., Anuar, A., Khairul Nizam, T., 2012. Terrain Slope Analyses between Terrestrial Laser Scanner and Airborne Laser Scanning, 2012 IEEE Control and System Graduate Research Colloquium. 